\title{
Risk avoidance: Graphs versus numbers
}

\author{
HANNAH FAYE CHUA, J. FRANK YATES, and PRITI SHAH \\ University of Michigan, Ann Arbor, Michigan
}

\begin{abstract}
There have long been speculations that graphical and numerical presentations of risk statistics differ in their impact on people's willingness to pursue actions that could harm or even kill them. But research has been unclear about the processes whereby the pictorial character of graphical displays per se might affect those risky decisions or even whether such effects actually occur. In two studies, we demonstrate that the pictorial nature of a graphical risk display can, indeed, increase risk avoidance. This increase is associated with a heightened impression of the riskiness of less safe alternatives. The results suggest that this picture-driven, intensified sense of riskiness, in turn, rests on two kinds of mechanisms: one cognitive, the other affective. Cognitively, pictorial presentations impose weaker upper bounds on people's internal representations of the chances that riskier alternatives will bring about actual harm. Affectively, pictures ignite stronger, more aversive negative associations with riskier options and their outcomes.
\end{abstract}

Since the 1940s (e.g., Preston \& Baratta, 1948), seemingly countless studies have sought to unravel the processes by which people choose among alternatives that differ in their riskiness. And that work has yielded considerable insights (see, e.g., Hastie, 2001). In many laboratory decision-making studies, to this day, the chances associated with the various outcomes that might result from risky alternatives are presented to participants numerically_for example, as probabilities that particular amounts of money will be gained or lost. In real life, such chances are, indeed, sometimes displayed to deciders that way, but sometimes they are represented differently-for instance, graphically. In a floor debate about motorcycle helmet laws, a legislator could show the numbers of helmeted and helmetless cyclists reduced to vegetative states in highway accidents over the past 5 years. Alternatively, the legislator could show a graph depicting the same statistics. Which display would be more persuasive in getting the legislator's colleagues to vote for stricter helmet requirements (the safer alternative)? More important from a theoretical perspective, how could we explain how logically equivalent pictorial and numerical descriptions might differentially affect choices among risky alternatives? Put another way, what would such effects imply about the nature of the fundamental processes responsible for risk avoidance behavior?

One definition of risk is that it is the possibility of loss (Yates \& Stone, 1992, p. 4). Risk increases both with

This research was supported in part by the University of Michigan Business School and a fellowship from the American Association of University Women. The authors are greatly appreciative of the excellent, helpful suggestions of three anonymous reviewers. We also thank Laura Klem for her invaluable data analytic assistance. Correspondence should be addressed to H. F. Chua, J. F. Yates, or P. Shah, Department of Psychology, University of Michigan, 525 East University Avenue, Ann Arbor, MI 48109-1109 (e-mail: hchua@umich.edu, jfyates@umich.edu, or priti@umich.edu). increases in the chances of potential losses and with increases in their severity. Thus, riding a motorcycle without a helmet would be riskier than otherwise if the probability of injury were greater or if the resulting injuries were more serious. There have long been speculations that graphs are especially effective tools for risk communication (e.g., Covello, Sandman, \& Slovic, 1988; Keeney \& von Winterfeldt, 1986). If true, this would imply that decisions predicated on graphical displays should be different from those based on other characterizations. Indeed, the expectation has been that the decisions should be better, although it is by no means obvious what better ought to mean.

Surprisingly, there was no empirical evidence bearing on speculations such as these before the experiments of Stone, Yates, and Parker (1997). In those experiments, the chances of loss (i.e., injuries) associated with various alternatives were displayed either numerically or graphically, in terms of stick figures, asterisks, bar graphs, or faces. The results indicated that graphical risk displays induce significantly greater risk avoidance than do numerical displays. Although Stone et al.'s (1997) experiments demonstrated the potential impact of graphs on risk avoidance, it remained a mystery precisely how such effects come about.

Then, on the basis of several experiments, Stone et al. (2003) concluded that the earlier findings of Stone et al. (1997) were probably due to a particular feature of the graphical displays that were employed-specifically, the prominence they gave to foreground, rather than background, risk information. Foreground information refers to the number of people harmed by a hazard, whereas background information characterizes the total number of people at risk of harm (or perhaps the number at risk who are, in fact, unharmed). Figure 1A shows one set of numerical displays like those presented to participants by Stone et al. (1997), who used the purchase of automobile tires as their risk-taking cover story. Here, we have 
A)

STANDARD TIRES

Cost: $\$ 225$ for 4

Annual Blowout Injury Risk (per 5,000,000 Ml drivers):

30 serious injuries

Foregro

oreground

Background
IMPROVED TIRES

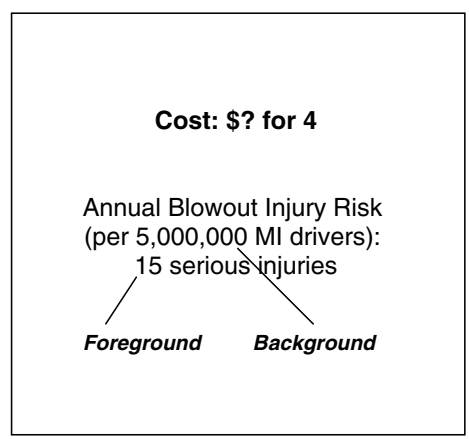

IMPROVED TIRES

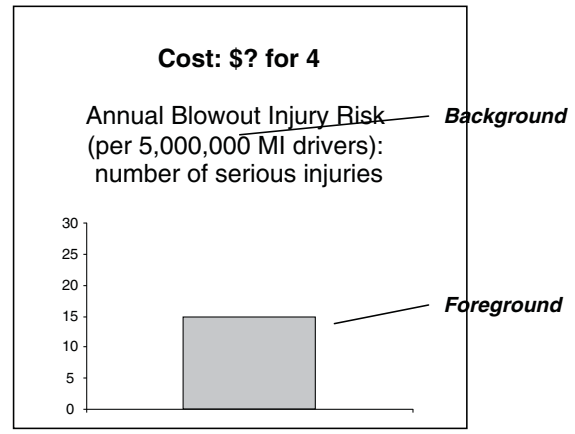

Figure 1. Displays adapted with permission from Figure 1(a), p. 247, and Figure 2(b), p. 250, of E. R. Stone, J. F. Yates, and A. M. Parker (1997). Effects of numerical and graphical displays on professed risk-taking behavior. Journal of Experimental Psychology: Applied, 3, 243-256, copyright 1997 by American Psychological Association. (A) Numerical displays; (B) bar graph displays. "Foreground" refers to number of people harmed by a hazard. "Background" refers to the total number of people at risk of harm.

explicitly labeled the foreground and background information. Consider the "Standard Tires" alternative. The background information indicates that 5,000,000 drivers are at risk. The foreground data indicate that 30 of those individuals can be expected to actually suffer harm (and thus 4,999,970 would remain unharmed). Observe that foreground and background elements of the risks attached to a given alternative - a particular kind of tire, in this instance-were displayed with equal physical prominence, embedded in the flow of a block of text.

Figure 1B shows bar graph representations of the same information. Note that the foreground facts, symbolized as bars that occupied significant portions of the visual field, are featured far more prominently than the background data. As is suggested by the phrase "Cost: $\$$ ? for 4," the participant's task was to state how much he or she would be willing to pay for a set of four "improved" tires, ones that put passengers at less risk. The participants were willing to pay significantly more for the safer productthat is, they were more risk avoidant-when the chances of harm were displayed graphically than when they were displayed numerically.

Stone et al.'s (2003) studies were, indeed, consistent with the conclusion that graphical effects on risk avoidance such as those demonstrated by Stone et al. (1997) are due to graphs' directing attention to foreground, rather than background, information. But there are many ways of focusing attention on foreground information at the expense of background information, including numerical techniques. One is the use of relative risk statistics (e.g., doubling disease risk), rather than incidence rates (e.g., 30 per 100,000 vs. 15 per 100,000; see Stone, Yates, $\&$ Parker, 1994). Thus, the question persists whether the pictorial character of graphs in and of itself is capable of affecting risk avoidance, beyond the well-established effects of foreground-background distinctions. More significantly, there is also the theoretical issue of how such effects occur, assuming that they do, since such explanations would have implications both for risky choice processing and for graph processing more generally. These were the questions pursued in the present research.

Unfortunately, previous scholarship on graphical displays has provided little guidance on the specific issues posed here. This is partly because the focus generally has been on other phenomena, such as comprehension and judgment accuracy, rather than on risk taking (e.g., Sanfey \& Hastie, 1998). And for the most part, the implications of the findings from the earlier graph literature for the present concerns have been ambiguous. Thus, although some studies have shown that information from graphical dis- 
plays is processed more rapidly or remembered more accurately than information from numerical displays, other studies have indicated exactly the opposite, depending on the format and task (Shah, Freedman, \& Vekiri, 2005). Moreover, it is unclear what such generic, elementary effects should imply for complex decision behaviors such as risk avoidance. With suitable assumptions, however, at least one previous conceptual analysis is consistent with a pictorial effect on risk avoidance.

Jarvenpaa (1990) proposed that in early, automatic perceptual processing, people's attention is drawn first to the visual contrasts that might exist in a display. Furthermore, the effects of this initial attention focus should persist during subsequent, deeper cognitive processing. In the present context, a pictorial display such as that in Figure 1B would channel early attention to the contrast in the chances of harm promised by the two alternatives. Then, in later cognitive analysis of all the relative merits of those options, more attention than otherwise would be devoted to that contrast, which favors the less risky option. That is, greater risk avoidance would result.

\section{STUDY 1}

The first aim of Study 1 was to establish whether the pictorial character of graphs can, in fact, reliably increase or decrease risk avoidance in and of itself, controlling for known foreground-background and gain-loss framing influences. In the event that the answer was positive, the more critical theoretical objective was to test the proposition that such effects are achieved via changes in people's risk magnitude perceptions, an effect that is at least compatible with the physical contrast attention proposal. That is, the intent was to determine whether a key element of graph processing in decision making under risk is that a pictorial rendering of loss statistics magnifies subjective impressions of contrasts in riskiness.

Figure 2A presents a set of bar graph displays used in the experiments of Stone et al. (2003), where toothpaste was the consumer product used in the cover story. Observe that, as in Figure 1B shown earlier, foreground information was represented much more prominently than background information. Figure 2B shows a different kind of graphi-
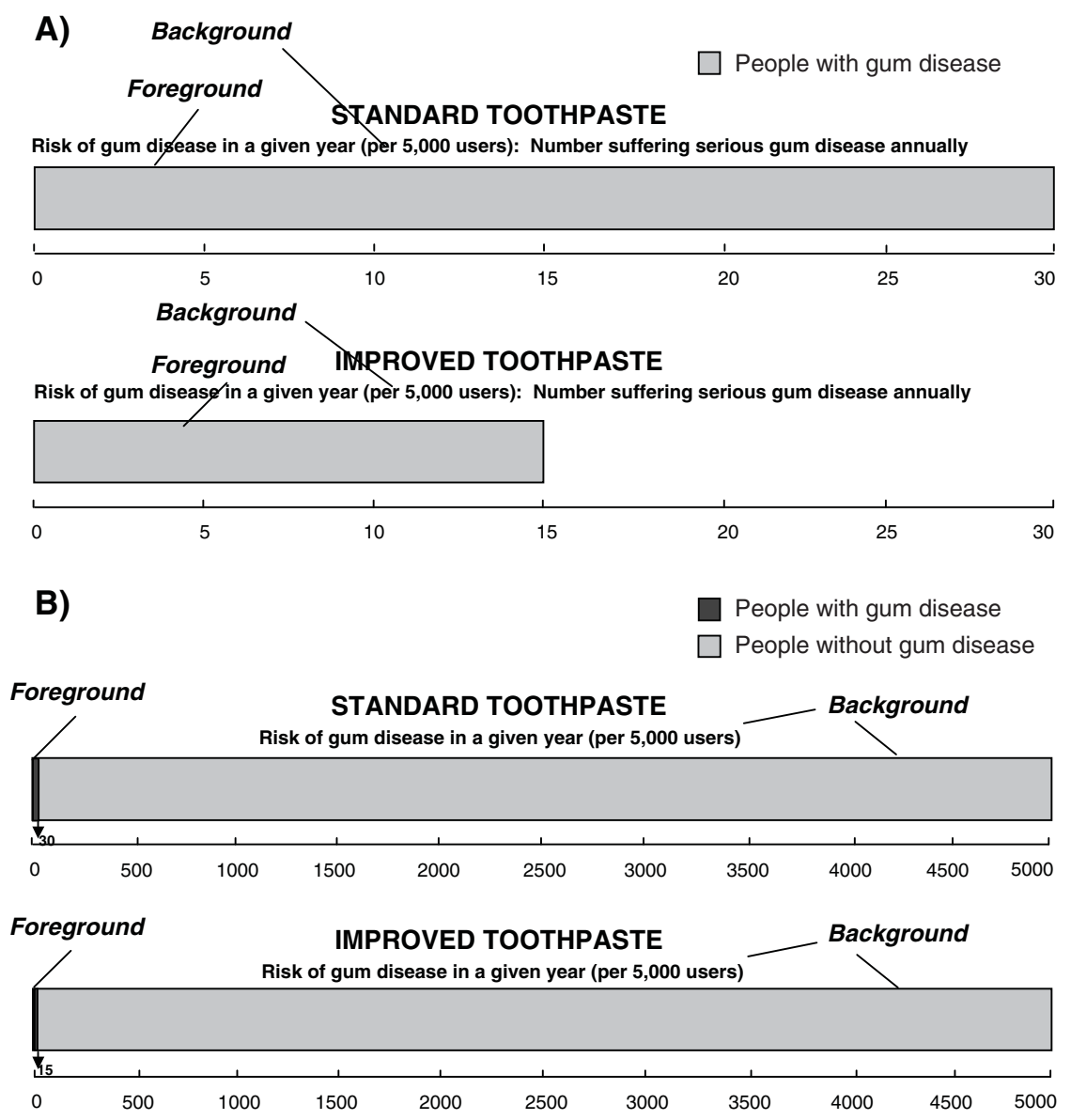

Figure 2. Displays adapted with permission from Figure 4, p. 28, in E. R. Stone et al. (2003), Foreground:background salience: Explaining the effects of graphical displays on risk avoidance. Organizational Behavior \& Human Decision Processes, 90, 19-36. Copyright 2003 by Elsevier. (A) Bar graph displays; (B) divided bar graph displays. "Foreground" refers to number of people harmed by a hazard. "Background" refers to the total number of people at risk of harm (or the number who are in fact unharmed). 
cal display employed by Stone et al. (2003), one depicting stacked or divided bar graphs. Note that, in contrast to the regular bar graphs, in the divided bar graphs, both the foreground and the background data were represented pictorially (and proportionately). Participants' risk avoidance was significantly weaker when likelihoods were displayed in the form of divided, rather than ordinary, bar graphs, and it was not significantly different from that induced by numerical displays of those risks. Hence, the interpretation of the results of Stone et al. (1997) as being due to foreground-background salience differences.

Given the comparisons by Stone et al. (2003) among numerical, bar graph, and divided bar graph displays, there was no incontestable effect of pictorial representation beyond its use as one particular device for focusing different amounts of attention on foreground versus background risk statistics. Drawing conclusions about such effects from the displays in Stone et al.'s (2003) experiments is further complicated by potential gain-loss framing influences. It is well known that people often respond very differently to alternatives that are described in terms of gains versus equivalent losses (Levin, Schneider, \& Gaeth, 1998). In gain framing, the consequences of an alternative are described positively (e.g., hamburger can be advertised as $70 \%$ lean). But in loss framing, those very same consequences are characterized negatively (e.g., 30\% fat). People have been shown to respond more strongly to alternatives in negative, rather than positive, frames (e.g., Meyerowitz \& Chaiken, 1987). Note that the bar graphs in Figure 2A pictorially depict only foreground information - that is, chances of loss (specifically, the likelihood of people's being harmed). In contrast, the divided bar graphs in Figure 2B pictorially describe both foreground and background data. The background information pertained to gains - that is, people unharmed by the pertinent hazard. The present study employed displays intended to clearly identify potential pictorial effects, controlling for gain-loss framing effects, since the existence of such effects had already been established.

\footnotetext{
Method

Participants. One hundred thirty-eight introductory psychology students (69 males and 69 females) at the University of Michigan served as volunteer participants in exchange for course credit, with 70 randomly assigned to the graphical display condition (described below) and the remaining 68 to the numerical display condition.

Materials and Procedure. Each participant was asked to respond to a scenario adapted from Stone et al. (2003; Stone et al., 1997). In that scenario, participants were told that there was a certain chance of developing periodontitis - that is, serious gum diseaseassociated with regularly using "standard toothpaste." The manufacturer was considering marketing a new brand of toothpaste, "improved toothpaste," which would be identical to standard toothpaste except that it would contain a new additive recently discovered to reduce gum disease risk. The participants were then given the price of standard toothpaste (\$2.29) and were asked how much they would be willing to pay for the safer product. As is shown in Figure 3, there were four different versions of the scenario, which differed only in how the chances of developing gum disease associated with each product were displayed. That information was presented either graphically or numerically (display type) and in either a gain or a loss frame (frame type).
}

In the loss frame, the risk information highlighted the number of people "suffering" from periodontitis. In the gain frame, the risk was described in terms of the number of people "free" from serious gum disease. In the numerical displays, only the numbers of users suffering or free from gum disease were given. In the graphical displays, we used bar graphs whose lengths represented the numbers of users suffering or free from gum disease. Actual numbers of users were indicated in the graphs, too. ${ }^{1}$ In all cases, the total number of toothpaste users at risk (5,000 users) was shown numerically. Note that in a given display, the facts that are represented pictorially or numerically are either foreground or background information only, not both. This permits a sharp separation of the effects of pictorial versus numerical displays from those of framing.

After the scenario-and-response sheets were returned to the experimenter, the participants completed a second questionnaire. The questionnaire included items in which the participants were asked about their perceptions of (1) initial risk, the degree of risk of periodontal disease associated with standard toothpaste (7-point scale); (2) risk reduction magnitude, the size of the reduction in gum disease risk resulting from using improved toothpaste, rather than standard toothpaste (7-point scale); (3) percentage of risk reduction, the percentage of reduction in gum disease risk resulting from using improved toothpaste instead of standard toothpaste; and (4) significance of risk reduction, the (personal, perceived) significance of the reduction in periodontal disease risk achieved by changing from standard to improved toothpaste.

\section{Results and Discussion}

Figure 4A shows the mean prices the participants were willing to pay for improved toothpaste in the various conditions. As is indicated, the prices reported by the participants presented with graphical displays were significantly higher than the prices stated by those exposed to the numerical displays $[F(1,134)=6.99, p=.009]$. (Tests on prices were performed on logarithmically transformed data, because the original data were skewed.) Also, in accord with expectations but of no central interest here, the participants in the loss framing conditions indicated higher prices for improved toothpaste than did those in the gain framing conditions $[F(1,134)=43.67, p<.001]$. The interaction of frame and display type was not significant $[F(1,134)=$ 1.16, n.s.]. Thus, it appears that the pictorial character of graphical displays, controlling for gain-loss framing effects, is indeed capable of affecting people's risk avoidance. In instances such as the present, pictorial representation should be expected to increase avoidance.

When viewing the stimuli as shown in Figure 3 (and especially with the benefit of hindsight; Slovic \& Fischhoff, 1977), the reader may be tempted to say that the observed effects are obvious. After all, one is then taking the perspective of the participants who actually experienced those effects. But it is not at all obvious why the effects occurred, which is the primary concern here. To shed light on the underlying processes, we regressed the participants' prices on various aspects of their perceptions of gum disease risk, as reported in the follow-up questionnaire.

The following was the regression model that was fitted to the data $\left[R^{2}=.297 ; F(4,130)=13.73, p<.001\right]$ :

$$
Y=\beta_{0}+\beta_{\mathrm{I}} X_{\mathrm{I}}+\beta_{\mathrm{R}} X_{\mathrm{R}}+\beta_{\mathrm{P}} X_{\mathrm{P}}+\beta_{\mathrm{S}} X_{\mathrm{S}},
$$

where $Y$ is the risk avoidance index (the participant's indication of the highest price he or she would be willing to 


\section{A) Graphical Display: Loss Frame}

\section{STANDARD TOOTHPASTE}

Risk of Gum Disease in a Given Year (Per 5,000 Users): Number of Users of STANDARD TOOTHPASTE Suffering From Serious Gum Disease Annually:

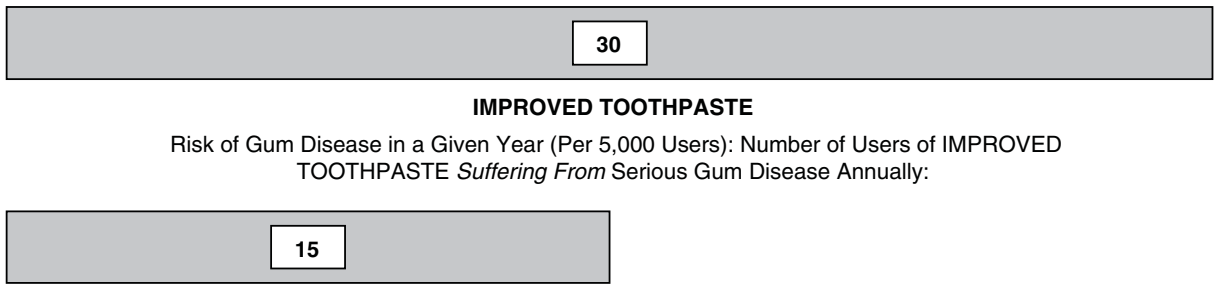

B) Graphical Display: Gain Frame

STANDARD TOOTHPASTE

Risk of Gum Disease in a Given Year (Per 5,000 Users): Number of Users of STANDARD TOOTHPASTE Free From Serious Gum Disease Annually:

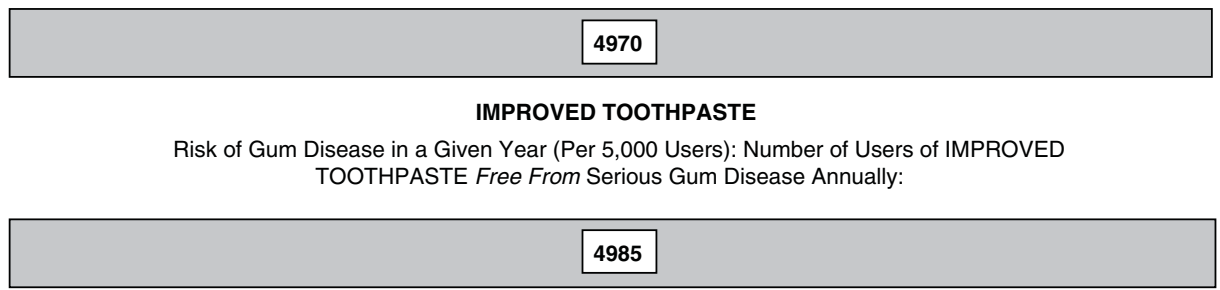

C) Numerical Display: Loss Frame

STANDARD TOOTHPASTE

Risk of Gum Disease in a Given Year (Per 5,000 Users): Number of Users of STANDARD TOOTHPASTE Suffering From Serious Gum Disease Annually:

30

IMPROVED TOOTHPASTE

Risk of Gum Disease in a Given Year (Per 5,000 Users): Number of Users of IMPROVED TOOTHPASTE Suffering From Serious Gum Disease Annually:

15

D) Numerical Display: Gain Frame

\section{STANDARD TOOTHPASTE}

Risk of Gum Disease in a Given Year (Per 5,000 Users): Number of Users of STANDARD TOOTHPASTE Free From Serious Gum Disease Annually:

4970

\section{IMPROVED TOOTHPASTE}

Risk of Gum Disease in a Given Year (Per 5,000 Users): Number of Users of IMPROVED TOOTHPASTE Free From Serious Gum Disease Annually:

4985

Figure 3. Displays used in Study 1: (A) graphical display with loss framing; (B) graphical display with gain framing; (C) numerical display with loss framing; (D) numerical display with gain framing.

pay for improved toothpaste), $\beta_{\mathrm{I}} X_{\mathrm{I}}$ is the index of perceived initial risk and its regression weight $\left(X_{\mathrm{I}}\right.$ is the participant's rating of the risk of periodontal disease associated with standard toothpaste), $\beta_{\mathrm{R}} X_{\mathrm{R}}$ is the index of perceived risk reduction magnitude and its regression weight $\left(X_{\mathrm{R}}\right.$ is the participant's rating of the magnitude of the reduction in gum disease risk resulting from using improved toothpaste rather than standard toothpaste, $\beta_{\mathrm{P}} X_{\mathrm{P}}$ is the percentage risk reduction index and its regression weight $\left(X_{\mathrm{P}}\right.$ was the participant's estimate of the percentage of reduction in gum disease risk achieved after switching from standard to improved toothpaste), and $\beta_{\mathrm{S}} X_{\mathrm{S}}$ is the index of the significance of risk reduction and its regression weight $\left(X_{\mathrm{S}}\right.$ is the participant's rating of the [perceived, personal] significance of the reduction of periodontal disease risk after changing from standard to improved toothpaste).

Only one of the four individual risk perception measures entered the regression significantly: the participants' ratings of the initial risk - that is, the riskiness of standard toothpaste [standardized $\beta_{\mathrm{I}}=.198 ; t(130)=2.05, p<$ .05]. [The remaining standardized coefficient estimates were the following: $\beta_{\mathrm{R}}=.213, t(130)=1.65, p=.10$; 


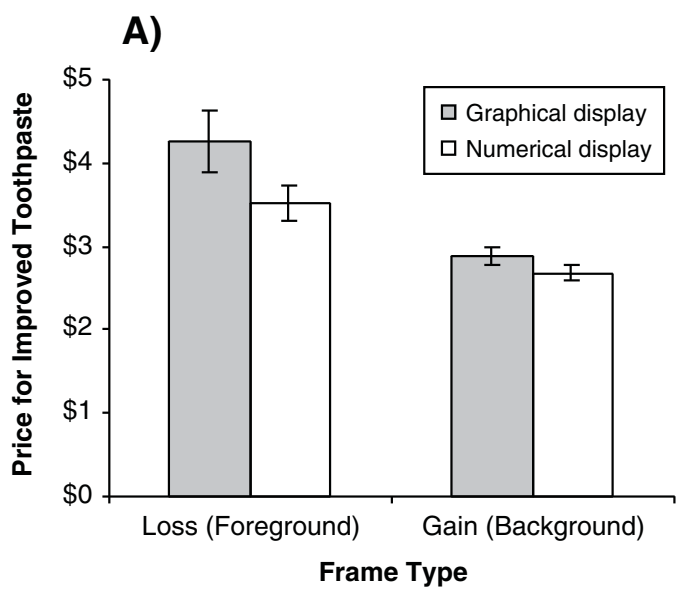

B)

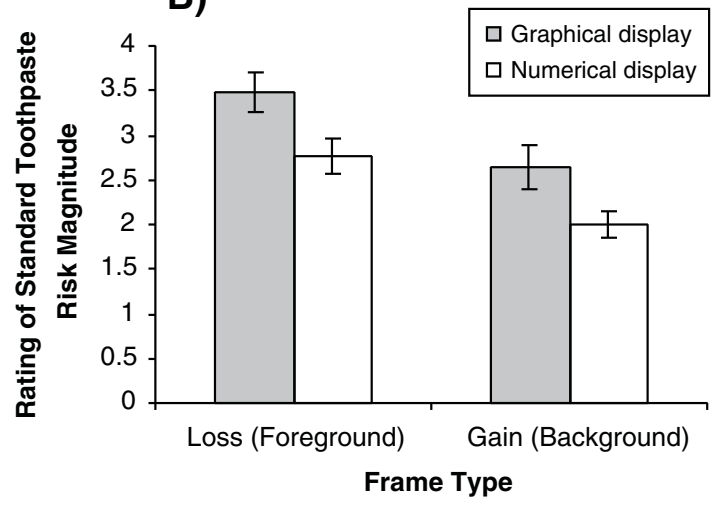

Figure 4. (A) Mean price the participants were willing to pay for improved toothpaste. (B) Mean ratings for the degree of risk of periodontal disease with standard toothpaste.

$\beta_{\mathrm{P}}=.076, t(130)=0.78$, n.s.; and $\beta_{\mathrm{S}}=.153, t(130)=$ 1.27 , n.s.] Table 1 shows the correlations among the various risk perception indices, which were clearly strongly related to one another, as well as to the risk avoidance measure. The high degree of collinearity implies that the specific values of the various coefficient estimates would likely vary substantially from one random sample to another. But it also makes clear that risk avoidance was strongly related to how individuals perceived the risk associated with the alternatives presented to them. Furthermore and importantly, as is shown in Figure 4B, graphical displays appeared to magnify the participants' perceptions of the riskiness of standard toothpaste $[F(1,134)=10.27$, $p=.002]$, and so did loss frames $[F(1,134)=13.98, p<$
.001], although the latter effect had no substantive interest here. (The interaction was nonsignificant.)

In sum, the data imply that pictorial representation is, in fact, capable of influencing risk avoidance; specifically, it intensifies such behavior. The results also implicate a mechanism by which this intensification occurs. In particular, pictures magnify the observer's sense that risky alternatives are too risky and, thus, perhaps should be rejected in favor of safer options.

\section{STUDY 2}

Study 1 demonstrated that one means by which graphical representation of risk statistics influences people's decisions among risky alternatives rests on induced perceptions of the risk magnitudes entailed in those options. But there persists the question of exactly how that induction occurs and whether there are additional routes by which graphs foster risk avoidance. In Study 2, evidence bearing on these processes was sought.

Figure 5 is useful for conceptualizing the theoretical analysis of how pictorial displays might affect risk avoidance. This attention priority model suggests that pictorial displays (0) trigger certain critical attention mechanisms (2), very likely including the ones suggested by Jarvenpaa (1990), as well as others to be discussed below. We propose that these mechanisms ultimately result in heightened risk avoidance (6) via either or both of two basic routes that were initially suggested by the nature of the risk construct.

Recall that risk, the possibility of loss, entails two core ideas (Yates \& Stone, 1992). The first is uncertainty, which in many instances is simply the chance of loss or harm of some sort. In the kinds of scenarios discussed so far, this might be, for example, the probability of developing gum disease. The second core idea is the magnitude and significance —objective and, often, personal —of that harm. In a gum disease scenario, this might entail the extent of disease an individual might experience and how distasteful—or not—such an occurrence would be for that particular person. It is worth noting that formal measures of risk typically entail multiplicative combinations of loss chances and indicators of how much those losses matter (see Yates \& Stone, 1992). For instance, concepts similar to expected loss involve combinations such as $p(\mathrm{~L}) \times$ $v(\mathrm{~L})$, where $p(\mathrm{~L})$ denotes the probability of a particular loss (e.g., the judged probability of developing gum disease) and $v(\mathrm{~L})$ represents the personal value one attaches

Table 1

Correlations Among Risk Avoidance and Risk Perception Indices, Study 1

\begin{tabular}{|c|c|c|c|c|c|}
\hline & $\begin{array}{l}\text { Risk Avoidance } \\
\text { Index }\end{array}$ & $\begin{array}{l}\text { Initial Risk } \\
\text { Index }\end{array}$ & $\begin{array}{l}\text { Risk Reduction } \\
\text { Magnitude Index }\end{array}$ & $\begin{array}{c}\text { Percentage of Risk } \\
\text { Reduction }\end{array}$ & $\begin{array}{c}\text { Significance of } \\
\text { Risk Reduction Index }\end{array}$ \\
\hline Risk avoidance index & - & & & & \\
\hline Initial risk index & $.44^{* *}$ & - & & & \\
\hline Risk reduction magnitude index & $.46^{* *}$ & $.55^{* *}$ & - & & \\
\hline Percentage of risk reduction & $.40^{* *}$ & $.55^{* *}$ & $.55^{* *}$ & - & \\
\hline Significance of risk reduction index & $.49^{* *}$ & $.60^{* *}$ & $.78^{* *}$ & $.60^{* *}$ & - \\
\hline
\end{tabular}

$$
{ }_{* *}^{*} p<.01 \text {. }
$$




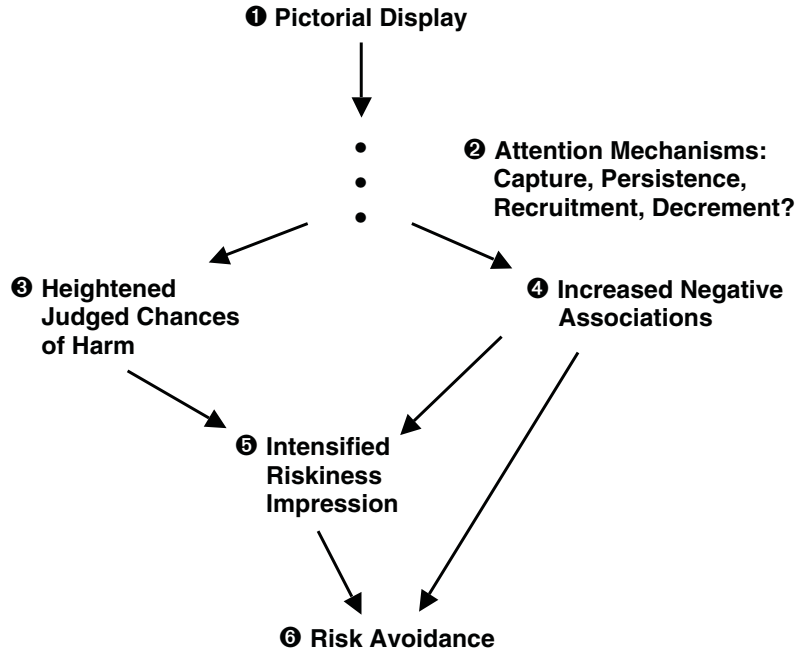

Figure 5. Elements of a proposed attention priority model of the processes by which pictorial displays of risk statistics might affect risk avoidance.

to that eventuality (e.g., the degree of distress the disease causes for the person afflicted). The results of Study 1 indicate that graphical displays intensify the impression that high-risk alternatives are significantly riskier than their competitors (6 in Figure 5). What is unsettled, however, is whether this intensification results from effects on the chances element of risk perception, the value element, or both. It is also unknown whether the systematic impact of pictorial displays on risk avoidance might not also arise partly from mechanisms distinct from risk perceptions. All these possibilities have some degree of theoretical plausibility and were addressed in Study 2.

First, consider the chances route to heightened impressions of riskiness, one that is essentially cognitive. There is reason to believe-as well as evidence-that cognitive representations of chances of harm tend to be simple, embodying the gist of external representations, not all the details (see Reyna \& Brainerd, 1991). Suppose, for instance, that an individual is presented with information on the chances of harm associated with two different products. That person's reasoning would likely turn on such beliefs as "Product A is much more hazardous than Product B," rather than on something like "Product A's injury rate is 48 out of 10,000 , whereas Product B's rate is 33 out of 10,000."

Imagine that a person sees a numerical display in which the injury rate for one alternative is clearly greater than that for another. The internal code in the cognitive representation is likely to be something like large difference. Now suppose that, instead, an equivalent graphical display captures that individual's attention. Again, the internal code is likely to be something coarse-perhaps, large difference. But large should be different in these two instances. In the numerical case, the meaning of large is tightly constrained by the actual numbers that were presented (e.g., 48/10,000 vs. 33/10,000). In contrast, in the case of graphs such as those studied here, the meaning of large is less constrained at the high, unbounded end. This is because the comparison is between pictures of, say, two bars of different lengths, whose meanings in terms of hazard severity are indefinite. (This assumes that the person does not explicitly and effortfully form a composite pictorial and numerical representation incorporating both bar lengths and scale values, which is unlikely to occur, per work by Kleinmuntz \& Schkade, 1993, as well as by Sanfey \& Hastie, 1998.) That is, the effective, perceived difference often should be larger in the graphical case, and accordingly, there should be greater risk avoidance.

Memory provides a window on cognitive representations of alternatives. Suppose that some individuals are shown numerical displays, whereas others see the same information conveyed graphically. And later, there is a request to recall the specific numerical values attached to those alternatives. Some respondents will accurately remember or estimate the actual numbers. Such accuracy should be especially common for those who had been exposed to numerical displays (Meyer, Shinar, \& Leiser, 1997), because their original internal representations often were themselves numerical. According to the previous arguments, in cases in which there are recall errors, those who have been presented with pictorial displays should make estimates that are higher than those reported by individuals who have seen numerical displays.

Now consider the value route to more intense senses of riskiness, which entails one particular kind of affective process. It is known that some aspects of visual displays (e.g., colors) influence affective responses (Hemphill, 1996; Levy, 1984; Valdez \& Mehrabian, 1994) and that these effects occur immediately and automatically. It is plausible that, in a similar fashion, pictorial risk displays ignite affective reactions manifested by, say, negative associations (4 in Figure 5) that are more intense than those induced by numerical displays. That is because, in comparisons like the ones of interest, (1) the obvious differences between the alternatives concern losses, (2) the pictures steer attention toward those differences (as has been shown by Jarvenpaa, 1990), and (3) people are known to be especially sensitive to losses (Kahneman \& Tversky, 1979). Thus, graphical displays would make potential harm feel worse, and hence, subjective riskiness should increase.

Finally, consider another, quite distinct means by which graphical displays might contribute to risk avoidance. Recent theorizing and evidence have suggested that affect sometimes has direct influences on people's risk taking (e.g., Finucane, Alhakami, Slovic, \& Johnson, 2000; Finucane, Peters, \& Slovic, 2003; Loewenstein, Weber, Hsee, $\&$ Welch, 2001). In so many words, the proposition is that risky alternatives tend to have negative affect attached to them as entire, undifferentiated entities. That affect would not be connected solely to their adverse outcomes, as is implicit in standard decision theoretic conceptions, such as expected utility theory (see Yates, 1990, chap. 9). The precise mechanisms by which this might occur are the subject of intense current study. They seem to be implicated in Damasio's (1994) somatic marker hypothesis and data bearing on that proposition (e.g., Bechara, Dama- 
sio, Tranel, \& Damasio, 1997). According to that view, over time, the normal human brain develops associations with hazardous, risky alternatives that tend to bias the person against choosing those options, largely outside of consciousness. Similar "hot" processes are implicated in attitudes that may or may not become active in a given instance, according to principles of accessibility that are gradually being elucidated (see Fazio, 1995). Indeed, as has been demonstrated in various studies, it seems that these emotion-focused processes have considerable functional value, such as making decision making easier and faster (Fazio, 1995, p. 262). So, as is suggested at 4 in Figure 5, if graphs increase negative associations with risky alternatives, this should more or less directly reduce the appeal of those alternatives.

In summary, Study 2 was intended to test the proposition that cognitive representations of the chances of harm (as reflected in memory performance) are one route by which graphs alter perceived differences in risk magnitude. It also investigated whether graphs increase negative associations to risky alternatives, which, in turn, contribute to risk avoidance directly, as well as through heightened risk impressions.

\section{Method}

Participants. One hundred fifty-five students in an introductory psychology course at the University of Michigan served as volunteer participants in this study, 83 in a graphical display condition (discussed below), with the remaining 72 in a numerical display condition.

Materials and Procedure. The participants completed several computer-administered tasks. Each participant was randomly assigned to respond to either a graphical or a numerical display of the scenario, this time involving tire purchases as the cover story, since one of our goals was to establish the generality of the effects in question. Figure 6 presents the displays used in the study. Those displays depict foreground information (chances of serious injuries resulting from tire blowouts) associated with using standard tires and improved tires. In all cases, the total number of tire users at risk of harm $(5,000,000$ users $)$ - that is, background information-was shown numerically, too.

Two risk avoidance measures were collected after the participants had read the scenarios. The first was price. The participants reported the highest amount that they would be willing to pay for a set of improved tires, given that a set of standard tires costs $\$ 325$. The second measure was a recommendation concerning the product. The participants indicated the degree to which they would be inclined to recommend that other people buy improved tires, rather than standard tires. Each participant chose a rating ranging from 1 (do not recommend) to 7 (strongly recommend).

After that, affective associations with the displays were assessed, using a semantic differential task. The participants were shown 10 pairs of words, one positive and one negative. For each pair, the participant indicated the number between 1 and 7 that best described the degree to which the "feeling" associated with the risky alternative (standard tires) was closer to that conveyed by one word versus the other. The word pairs were: good-bad*, boredom-excitement, safety-danger*, smart-stupid*, cold-hot, love-hate*, sick-healthy, pretty-ugly*, weak-strong, and happy-sad*, where the ratings for pairs marked by asterisks were later reverse scored.

\section{A)}

\section{STANDARD TIRES}

Annual Blowout Injury Risk (per 5,000,000 MI drivers): Number of Passengers Suffering Serious Injuries in Cars with STANDARD TIRES:

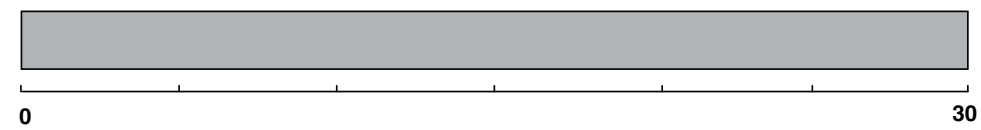

IMPROVED TIRES

Annual Blowout Injury Risk (per 5,000,000 MI drivers): Number of Passengers Suffering Serious Injuries in Cars with IMPROVED TIRES:

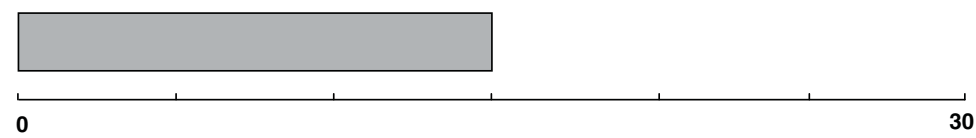

B)

STANDARD TIRES

Annual Blowout Injury Risk (per 5,000,000 MI drivers): Number of Passengers Suffering Serious Injuries in Cars with STANDARD TIRES:

30

\section{IMPROVED TIRES}

Annual Blowout Injury Risk (per 5,000,000 MI drivers): Number of Passengers Suffering Serious Injuries in Cars with IMPROVED TIRES:

Figure 6. Displays used in Study 2: (A) graphical; (B) numerical. 
In their final task, the participants were asked several incidental memory questions concerning chances of loss: (1) background information ("What is the population of MI [Michigan] drivers they base the annual blowout injury risk on?"), (2) foreground information associated with standard tires ("How many passengers suffer injuries annually in cars with standard tires per the population of MI drivers mentioned?"), and (3) foreground information associated with improved tires ("How many passengers suffer from serious injuries annually in cars with improved tires per the population of MI drivers mentioned?").

\section{Results and Discussion}

The participants presented with graphical displays reported significantly higher ratings of their willingness to recommend that others buy improved tires, rather than standard tires, than did those exposed to numerical displays $[M \mathrm{~s}=5.40$ vs. 5.06, respectively; $t(153)=2.37$, $p=.019]$. The participants who saw graphical displays were also somewhat more willing to pay a high price for a set of four improved tires than were those presented with numerical displays ( $M \mathrm{~s}=\$ 436.41$ vs. $\$ 431.81$ ), although this price difference was not statistically significant $(t<1$, n.s.). These results again indicate support for the conclusion that pictorial displays are indeed capable of increasing risk avoidance. ${ }^{2}$

Recall of risk statistics. Table 2 shows the percentages of participants in the various conditions who accurately recalled the risk statistics presented to them. As was expected, the participants who viewed graphs were relatively less accurate in remembering the precise numbers of people suffering serious injuries using standard tires $\left[\chi^{2}(1\right.$, $N=155)=15.58, p<.001]$ and improved tires $\left[\chi^{2}(1\right.$, $N=155)=21.78, p<.001]$. Accuracy of memory for background information-the reference number of people exposed to the risk of serious injury $(5,000,000)$ - was comparable for the participants who saw graphical or numerical displays $\left[\chi^{2}(1, N=155)=1.80\right.$, n.s. $]$. Thus, there is no reason to believe that the participants assigned to the comparison groups differed in memory ability a priori. Nor is there reason to conclude that pictures distracted attention from numerical information generally.

Table 3 presents the medians of the risk statistics recalled by the participants who failed to correctly report the actual risk indicators shown to them earlier. Those exposed to graphical displays reported greater injury rates associated with standard tires than did those presented with numerical displays (Mann-Whitney $U=59.5, z=-2.17$, $p=.03$ ). Reported risk statistics for improved tires were not different among the participants who saw graphical or numerical displays (Mann-Whitney $U=72.0, z=-.36$, n.s.). Differences in risk statistics reported for standard and improved tires were also computed and compared for participants who recalled at least one of the risk statistics incorrectly. The participants who saw graphical displays reported larger injury rate differences between standard and improved tires than did those who viewed numerical displays (Mann-Whitney $U=51.0, z=-2.30, p=.022$ ). These results are in accord with the hypothesis that those who saw pictorial displays constructed internal cognitive representations in which especially large injury chances were associated with standard tires, the riskier option. The data indicated that the participants in the graphical condition also encoded the differences in injury rates for the two alternatives as large, in accord with an induction of greater risk avoidance. Thus, in summary, these data indicate that at least part of the mechanism by which graphs intensify the impression of riskiness is a cognitive one entailing heightened chances of harm (3 in Figure 5).

Affect ratings. An affect scale was created on the basis of the participants' mean responses to the 10 pairs of words in the affective associations task (Cronbach's $\alpha=$ .83). Overall, the participants who saw pictorial displays reported more negative ratings toward standard tires, the riskier alternative, than did those who viewed numerical displays $[M \mathrm{~s}=3.30$ vs. $3.64 ; t(153)=2.29, p=.023$; the lower the rating, the more negative the association with standard tires]. Affect scores were also correlated with the participants' recommendation ratings. The more negatively the participants rated standard tires, the more willing they were to recommend that others buy improved tires, the safer alternative $(r=-.242, p=.003)$. These findings are in accord with the conclusion that an additional route by which graphs heighten the impression of riskiness, thereby encouraging risk avoidance, involves making potential harm feel worse (4 in Figure 5).

Path analyses. Post hoc path analyses were used to test for direct, as well as indirect, influences on risk avoidance. The analyses were implemented using Amos 5 software (Amos Development Corporation). Figure 7 shows the model that was tested, which was an instantiation of the lower portion of the attention priority model sketched in Figure 5. A multigroup model with no cross-group constraints was tested for both the graphical and the numerical display groups and was not rejected, indicating that the model form did not differ between the groups $\left[\chi^{2}(2\right.$, $N=155)=1.59, p>.05 ; \mathrm{CFI}=1.00, \mathrm{RMSEA}=.02$ (low-confidence interval $=0$, high-confidence inter-

Table 2

Actual Values of Risk Statistics and Percentages of Participants With Accurate Recall of Those Statistics, Study 2

\begin{tabular}{lccc}
\hline \multicolumn{1}{c}{ Statistic } & Accurate Recall by Display Type \\
\cline { 2 - 4 } & Actual Value & Graphical (\%) & Numerical (\%) \\
\hline Number of serious injuries: Standard tires & 30 & 59.0 & $87.5^{* *}$ \\
Number of serious injuries: Improved tires & 15 & 56.7 & $90.3^{* *}$ \\
Reference number of drivers & $5,000,000$ & 43.4 & 54.2 \\
\hline
\end{tabular}

${ }^{* *} p<.001$, graphical versus numerical. 
Table 3

Actual Values of Risk Statistics and Medians of Those Statistics

Reported by Participants With Erroneous Recall, Study 2

\begin{tabular}{|c|c|c|c|}
\hline \multirow[b]{2}{*}{ Statistic } & \multirow[b]{2}{*}{ Actual Value } & \multicolumn{2}{|c|}{ Reports by Display Type } \\
\hline & & Graphical & Numerical \\
\hline Number of serious injuries: standard tires ${ }^{\mathrm{a}}$ & 30 & 300 & $25^{*}$ \\
\hline Number of serious injuries: improved tires ${ }^{b}$ & 15 & 42 & 30 \\
\hline \multicolumn{4}{|l|}{ Difference in number of serious injuries } \\
\hline from standard vs. improved tires ${ }^{c}$ & 15 & 77 & $2^{*}$ \\
\hline
\end{tabular}

val $=0.15$, PCLOSE $=.57)] .{ }^{3}$ We then tested a fully constrained model in which the direct paths of the model were constrained to be the same for both groups. The fully constrained model produced a less than desirable fit to the observed data $\left[\chi^{2}(6, N=155)=20.38, p=.002\right]$. Next, we compared the goodness of fit of the constrained model with that of the unconstrained model. Goodness of fit for the constrained model was significantly worse than that for the corresponding unconstrained model; the $\chi^{2}$ difference was $18.80(d f=4, p<.001)$. This suggests that the path coefficients differ for the graphical display and the numerical display groups. The nature of the differences is apparent in the parameter estimates shown in Figure 7. Of particular interest, the links from affect to risk perceptions and recommendations were generally stronger for the graphical case. Moreover, in that circumstance, it appears that affect influences risk-taking recommendations directly, as well as through risk perceptions, consistent with recent proposals (e.g., Finucane et al., 2000).

\section{GENERAL DISCUSSION}

One essential question this research addressed was whether the pictorial character of graphical displays is capable of influencing people's risk avoidance behavior. The data indicated that the answer is yes. People appear to regard less risky alternatives more positively (e.g., in their willingness to recommend or pay for them) when risk statistics are rendered pictorially, rather than numerically. These effects seem to occur even when there are controls for previously established foreground-background salience and gain-loss framing influences.

More important from a theoretical perspective, to the extent that correlational studies can, the present studies also suggested details of the mechanisms by which graphical depictions of risk statistics induce greater risk avoidance than do numerical characterizations. Thus, these studies support the attention priority model outlined in Figure 5. First, such depictions amplify the impression that riskier alternatives are substantially riskier than competing alternatives. Second, this amplification is achieved through both elements of the risk construct: the chances of potential harm from a hazard, as well as the evaluative impact of that harm. That is, the kinds of graphs studied here induce a cognitive effect, reflected in memory data, whereby the likelihood of an adverse outcome appears greater than otherwise. These graphs also appear to enhance the impression of riskiness by igniting negative associations that should make anticipated adverse outcomes feel especially bad. Finally, the data suggest that there is another, more direct affective means by which graphs encourage risk avoidance, one that is not mediated by risk impressions. According to this account (in accord with other evidence accumulating in the literature), graphically induced negative associations seem to become attached to riskier alternatives in a generalized, undifferentiated way, making them less appealing on the whole.

The present findings are useful. Yet there remain significant challenges for future studies - most notably, ones localized at the "Attention Mechanisms" point in the attention priority model shown in Figure 5. Recall that Jarvenpaa's (1990) attention data have been the sole finding in previous graph research that has seemed to buttress an expectation that pictorial representations of risk statistics should yield increased risk avoidance. The next task is to establish the precise nature of the operative attention processes. There are at least two major candidate hypotheses concerning the details.

First, it seems entirely plausible (and consistent with previous evidence; e.g., Mayer, Heiser, \& Lonn, 2001) that physical contrasts in pictorial displays of the chances of harm associated with less risky and more risky alternatives capture the person's attention early. But this alone is insufficient to explain why, for instance, the result should be heightened final judgments of the chances of harm from riskier alternatives. Another feature of Jarvenpaa's (1990) proposal might provide the link. The idea is that early attention to a particular aspect of a display results in increased attention to that element in later cognitive processing. Part of this processing might entail the recruitment of arguments bearing on the relative chances of harm associated with the alternatives at hand. More time devoted to the recruitment of arguments consistent with the initially perceived visual display would, indeed, seem to favor the kinds of judgment effects documented here. Such a process would appear to facilitate the increased negative associations we observed, too. Thus, suppose that the observer has an early impression that the riskier of two options is much riskier. Then, if more time is spent in that "biased" state, part of that time plausibly could be spent activating a wider set of negative associations with the riskier option-quite likely, in an automatic, noncon- 
A) Graphical Displays

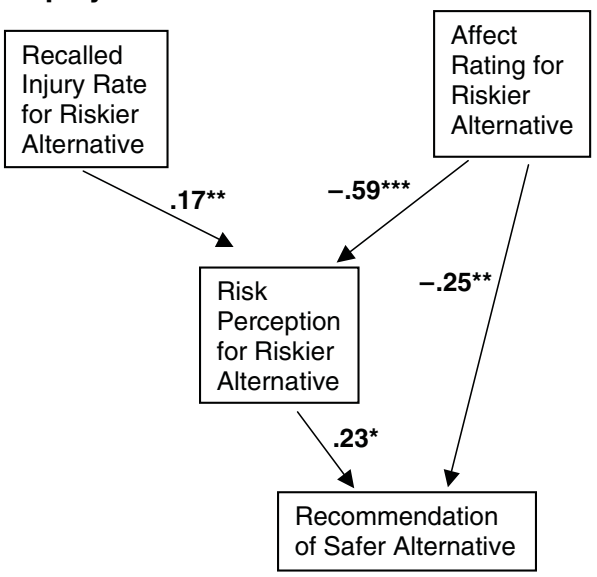

B) Numerical Displays

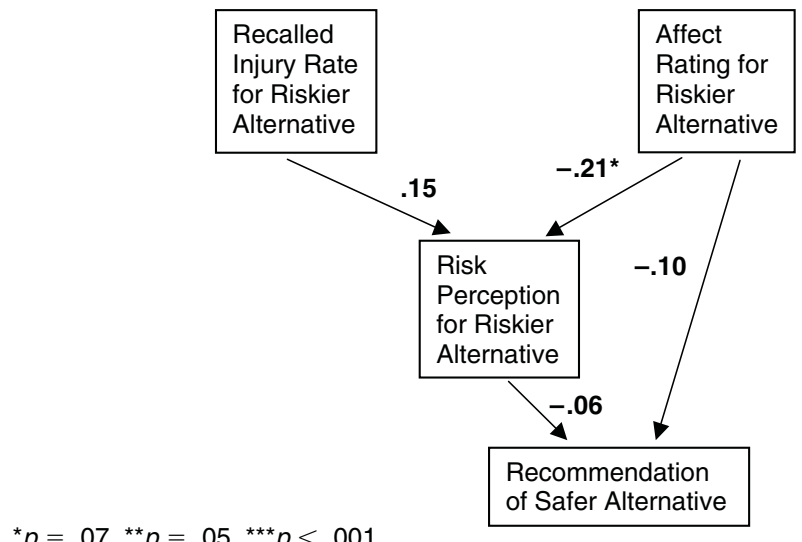

Figure 7. Path models with standardized coefficients for Study 2, given (A) graphical and (B) numerical displays. "Recalled Injury Rate for Riskier Alternative" pertains to the recalled risk statistics for standard tires. Data were recoded for the path analyses. Recalled injury rates that were equal to or less than 30 were assigned a value of 0 and recall values that were greater than 30 were assigned a value of 1. "Affect Rating for Riskier Alternative" pertains to the mean affect scores for standard tires (lower scores indicate more negative associations to standard tires; 7-point scale). "Risk Perception for Riskier Alternative" refers to the perceived initial risk of serious injury associated with standard tires (7-point scale). "Recommendation of Safer Alternative" was represented by the participants' ratings of how strongly they would recommend that others buy improved tires, rather than standard tires (7-point scale).

scious way. Such a mechanism is entirely consonant with recent striking evidence that when, for whatever (even arbitrary) reason, deciders are initially disposed toward one particular alternative, over time they restructure their mental representations of the alternatives so that these representations are increasingly supportive of that initially favored option (Simon, Krawczyk, \& Holyoak, 2004).

Another possible mechanism rests on the notion of attention decrement, which has been implicated in a variety of judgment phenomena, such as primacy effects in contingency assessment (e.g., Yates \& Curley, 1986). Accord- ing to this view, after an initial assessment based on early evidence, a person attenuates his or her attention to all the evidence encountered subsequently. In the present context, after a pictorial display creates a first impression that the chances of harm from a risky alternative are especially high, the judgment process would essentially end. As in the case of Jarvenpaa's (1990) increased attention idea, attention decrement is not guaranteed either. It is known that there are conditions under which it does not occur (Hogarth \& Einhorn, 1992). Clearly, the question of which attention mechanisms do indeed contribute to graphical display effects on harm likelihood judgments and on negative associations can only be settled empirically.

\section{REFERENCES}

Bechara, A., Damasio, H., Tranel, D., \& Damasio, A. R. (1997). Deciding advantageously before knowing the advantageous strategy. Science, 275, 1293-1295.

Covello, V. T., Sandman, P. M., \& Slovic, P. (1988). Risk communication, risk statistics and risk comparisons: A manual for plant managers. Washington, DC: Chemical Manufacturers Association.

Damasio, A. R. (1994). Descartes' error: Emotion, reason, and the human brain. New York: Putnam.

FAZIO, R. H. (1995). Attitudes as object-evaluation associations: Determinants, consequences, and correlates of attitude accessibility. In R. E. Petty \& J. A. Krosnick (Eds.), Attitude strength: Antecedents and consequences (pp. 247-282). Mahwah, NJ: Erlbaum.

Finucane, M. L., Alhakami, A., Slovic, P., \& Johnson, S. M. (2000). The affect heuristic in judgments of risks and benefits. Journal of Behavioral Decision Making, 13, 1-17.

Finucane, M. L., Peters, E., \& Slovic, P. (2003). Judgment and decision making: The dance of affect and reason. In S. L. Schneider \& J. Shanteau (Eds.), Emerging perspectives on judgment and decision research (pp. 327-364). New York: Cambridge University Press.

Hastie, R. (2001). Problems for judgment and decision making. Annual Review of Psychology, 52, 653-683.

Hemphill, M. (1996). A note on adults' color-emotion associations. Journal of Genetic Psychology, 157, 275-280.

Hogarth, R. M., \& EINHORN, H. J. (1992). Order effects in belief updating: The belief-adjustment model. Cognitive Psychology, 24, 1-55.

JARVENPAA, S. L. (1990). Graphic displays in decision making: The visual salience effect. Journal of Behavioral Decision Making, 3, 247-262.

Kahneman, D., \& Tversky, A. (1979). Prospect theory: An analysis of choice under risk. Econometrica, 47, 263-291.

KEENEY, R. L., \& VON WinterfeldT, D. (1986). Improving risk communication. Risk Analysis, 6, 417-424.

Kleinmuntz, D. N., \& SchKade, D. A. (1993). Information displays and decision processes. Psychological Science, 4, 221-227.

KLINE, R. B. (2005). Principles and practice of structural equation modeling (2nd ed.). New York: Guilford.

Levin, I. P., Schneider, S. L., \& Gaeth, G. J. (1998). All frames are not created equal: A typology and critical analysis of framing effects. Organizational Behavior \& Human Decision Processes, 76, 149-188.

LEVY, B. I. (1984). Research into the psychological meaning of color. American Journal of Art Therapy, 23, 58-62.

Loewenstein, G. F., Weber, E. U., Hsee, C. K., \& Welch, N. (2001). Risk as feelings. Psychological Bulletin, 127, 267-286.

Mayer, R. E., Heiser, J., \& Lonn, S. (2001). Cognitive constraints on multimedia learning: When presenting more material results in less understanding. Journal of Educational Psychology, 93, 187-198.

Meyer, J., Shinar, D., \& Leiser, D. (1997). Multiple factors that determine performance with tables and graphs. Human Factors, 39, 268-286.

Meyerowitz, B. E., \& Chaiken, S. (1987). The effect of message framing on breast self-examination attitudes, intentions, and behavior. Journal of Personality \& Social Psychology, 52, 500-510.

Preston, M. G., \& Baratta, P. (1948). An experimental study of the 
auction-value of an uncertain outcome. American Journal of Psychology, 61, 183-193.

ReYNA, V. F., \& Brainerd, C. J. (1991). Fuzzy-trace theory and framing effects in choice: Gist extraction, truncation, and conversion. Journal of Behavioral Decision Making, 4, 249-262.

Sanfey, A., \& Hastie, R. (1998). Does evidence presentation format affect judgment? An experimental evaluation of displays of data for judgments. Psychological Science, 9, 99-103.

Shah, P., Freedman, E., \& Vekiri, I. (2005). The comprehension of quantitative information in graphical displays. In P. Shah \& A. Miyake (Eds.), The Cambridge handbook of visuospatial thinking (pp. 426476). New York: Cambridge University Press.

Simon, D., KrawczyK, D. C., \& Holyoak, K. J. (2004). Construction of preferences by constraint satisfaction. Psychological Science, 15, 331-336.

SLOVIC, P., \& FischHoff, B. (1977). On the psychology of experimental surprises. Journal of Experimental Psychology: Human Perception \& Performance, 3, 544-551.

Stone, E. R., Sieck, W. R., Bull, B. E., Yates, J. F., Parks, S. C., \& RusH, C. J. (2003). Foreground:background salience: Explaining the effects of graphical displays on risk avoidance. Organizational Behavior \& Human Decision Processes, 90, 19-36.

Stone, E. R., Yates, J. F., \& Parker, A. M. (1994). Risk communication: Absolute versus relative expressions of low-probability risks. Organizational Behavior \& Human Decision Processes, 60, 387-408.

Stone, E. R., Yates, J. F., \& PARKer, A. M. (1997). Effects of numerical and graphical displays on professed risk-taking behavior. Journal of Experimental Psychology: Applied, 3, 243-256.
Valdez, P., \& Mehrabian, A. (1994). Effects of color on emotions. Journal of Experimental Psychology: General, 123, 394-409.

YATES, J. F. (1990). Judgment and decision making. Englewood Cliffs, NJ: Prentice Hall.

Yates, J. F., \& Curley, S. P. (1986). Contingency judgment: Primacy effects and attention decrement. Acta Psychologica, 62, 293-302.

Yates, J. F., \& Stone, E. R. (1992). The risk construct. In J. F. Yates (Ed.), Risk-taking behavior (pp. 1-25). Chichester, U.K.: Wiley.

\section{NOTES}

1. The data in Sanfey and Hastie (1998) suggest that such redundancy has little, if any, influence on participants' behavior.

2. The fact that the price difference was not statistically significant in this instance was puzzling. Yet it does not seem crucial, since significant differentials have been observed with the same cover story in previous experiments (e.g., Stone, Yates, \& Parker, 1997).

3. Three fit indices are reported: the chi-square tests of the fit of model to the data; the comparative fit index $(\mathrm{CFI})$, representing the relative improvement over an alternative model with no relations among the variables; and the root mean square error of approximation (RMSEA), indicating the difference between predicted and observed covariances (for a discussion of these indices, see Kline, 2005).

(Manuscript received August 20, 2004; revision accepted for publication March 3, 2005.) 